\title{
Effect of a nutritional intervention promoting the Mediterranean food pattern on electrophoretic characteristics of low-density lipoprotein particles in healthy women from the Québec City metropolitan area
}

\author{
Julie Goulet, Benoît Lamarche, Amélie Charest, Geneviève Nadeau, Annie Lapointe, Sophie Desroches \\ and Simone Lemieux* \\ Institute of Nutraceuticals and Functional Foods, 2440 Hochelaga Blvd, Laval University, Québec, Québec, Canada, G1K $7 P 4$
}

(Received 19 December 2003 - Revised 8 April 2004 - Accepted 15 April 2004)

\begin{abstract}
The objective of the present study was to evaluate the effect of a nutritional intervention promoting the Mediterranean food pattern in free-living conditions on LDL electrophoretic characteristics in a group of seventy-one healthy women, aged between 30 and 65 years. The 12-week nutritional intervention consisted of two courses on nutrition and seven individual sessions with a dietitian. The first course provided information on the Mediterranean food pattern and the second was a cooking lesson. LDL peak particle diameter (LDL-PPD) and cholesterol levels in small (LDL-cholesterol $<255 \AA$ ) and large LDL fractions (LDL-cholesterol $>260 \AA$ ) were obtained by $2-16 \%$ polyacrylamide gel electrophoresis of whole plasma. The sample was divided on the basis of baseline LDL-PPD using tertiles of the distribution (258.4 $\AA$ and 260.0 $)$ ). Among the total sample of women, no significant change in LDL-PPD was observed in response to the nutritional intervention. However, subjects who at baseline were in the first tertile of the LDL-PPD distribution $(<258.4 \AA)$ showed a significant increase in LDL-PPD and in the proportion of LDL $\%>260 \AA$ in response to the 12 -week nutritional intervention $(P<0 \cdot 05)$. In contrast, LDL-PPD decreased significantly $(P=0 \cdot 007)$ among women with large LDL particles at baseline (LDL-PPD $>260 \AA)$ while the proportion of LDL $\%<255 \AA$ and of LDL $\%_{>260 \AA}$ remained unchanged. To conclude, changes in the food pattern, in response to a nutritional intervention promoting the Mediterranean food pattern, were accompanied by beneficial modifications in LDL electrophoretic characteristics in women who were characterised at baseline by smaller LDL particles.
\end{abstract}

Mediterranean diet: Low-density lipoprotein peak particle diameter: Plasma lipids: Nutritional intervention

Interest in the Mediterranean food pattern can be partly explained by its effects on mortality and incidence of CHD. In fact, results of the Seven Countries Study which have demonstrated that the mortality rate from CHD was two- to three-fold lower in Southern Europe than in Northern Europe or in the USA have clearly contributed to the increased interest in the Mediterranean food pattern (Keys et al. 1986). In addition, more recent studies in men with coronary artery disease have indicated that the Mediterranean diet decreases the recurrence of CHD events by more than $50 \%$ compared with the usual prudent Western diet (de Lorgeril et al. 1994, 1999; Renaud et al. 1995). The recurrence of coronary events in the latter study was not associated with any modifications in the basic plasma lipid profile such as total cholesterol, LDL-cholesterol (LDL-C), and HDL-cholesterol (HDL-C) concentrations.

It is well known that many individuals with an apparently adequate plasma lipid profile, as determined by their LDL-C concentrations, develop CHD. Examination of plasma lipid characteristics not traditionally measured, for example LDL peak particle diameter (LDL-PPD) and LDL particles distribution, may provide important information that may contribute to a more refined prediction of CHD risk. Small, dense LDL particles appear to be more atherogenic than larger, less dense LDL particles (Austin et al. 1988). It has been shown recently that LDL-PPD did not appear as the most potent predictor of coronary artery disease risk associated with the small dense LDL phenotype in men of the Québec Cardiovascular Study. In contrast, an increased concentration of cholesterol in the LDL fraction with a diameter $<255 \AA$ (LDL-C $<255 \AA$ ) was associated with a four-fold elevation in CHD risk, independently of other lipid risk factors such as LDL-C, HDL-C, triacylglycerols (TG), lipoprotein (a) concentrations and LDL-PPD (St-Pierre et al. 2001).

Only limited data exist so far on the relationship between dietary factors and LDL size phenotype. It has been demonstrated that unsaturated fatty acids similarly reduce LDL particle size (Kratz et al. 2002), as do trans

\footnotetext{
Abbreviations: FFQ, food-frequency questionnaire; HDL-C, HDL-cholesterol; LDL-C, LDL-cholesterol; LDL-PPD, LDL peak particle diameter; SFA, saturated fatty acids; TG, triacylglycerols.

* Corresponding author: Dr Simone Lemieux, fax +1 418656 5877, email Simone.Lemieux@aln.ulaval.ca
} 
fatty acids (Mauger et al. 2003), while saturated fatty acids (SFA) increase LDL-PPD (Dreon et al. 1998). In contrast, some studies conducted in healthy men and women observed that neither type of dietary fat affected LDL size (Pedersen et al. 2000; Rivellese et al. 2003) but that a high-SFA diet was associated with higher LDL-C and TG concentrations (Rivellese et al. 2003). Moreover, it has been demonstrated that $n$-3 PUFA supplementation increases LDL-PPD in men and women (Tinker et al. 1999; Mori et al. 2000). A significant reduction in the mass of medium and small LDL size subfractions has been observed in healthy men characterised by smaller LDL particles compared with those with larger LDL particles when changing from a high-fat to a low-fat diet (Krauss \& Dreon, 1995). These results indicate that individuals with small, dense LDL particles may benefit more from dietary modifications than individuals presenting larger particles. However, Archer et al. (2003) showed in a well-controlled nutritional intervention conducted under ad libitum conditions that a high-carbohydrate diet or a high-MUFA diet, when associated with weight loss, have no deleterious effect on LDL electrophoretic characteristics in healthy men.

Our objective was to study the impact of a nutritional intervention promoting the Mediterranean food pattern in free-living conditions on LDL-PPD, an issue that has yet to be documented. In order to reach this objective, a group of seventy-one healthy women from the Québec City metropolitan area, aged between 30 and 65 years, and examined at 0,6 and 12 weeks of a nutritional intervention were studied.

\section{Subjects and methods}

Seventy-seven women from the Québec City metropolitan area were recruited through the Laval University newspaper during the summer of 2001. Women included in the study were aged between 30 and 65 years. To be eligible, women had to be free from metabolic disorders requiring treatment, to have a stable body weight for at least 3 months before the start of the study and to be in charge of food purchases and meal preparation most of the time. Only women with a diet concordant with the usual Canadian food pattern were included in the study. One hundred and twenty-six women were invited to a screening visit for an evaluation of their food habits. Among this initial group of women, ninety-four were found to be eligible according to the inclusion criteria. Seventy-seven women signed the informed consent form, which had been approved by ethics committees of Laval University. Three women left the study for personal reasons. Three other participants did not complete all $3 \mathrm{~d}$ food records or the food-frequency questionnaires (FFQ); therefore, seventy-one women were included in the analyses.

\section{Intervention}

The methodology of the nutritional intervention has been described previously (Goulet et al. 2003). Briefly, the study was conducted in two phases. Each phase was conducted using a similar 12-week intervention design. The first phase started in August 2001 and the second began in January 2002. The intervention included two group sessions with eight participants per group. During the first group session the registered dietitian explained the major principles of the Mediterranean diet and the health benefits associated with this food pattern. At 4 weeks after the beginning of the intervention, the subjects were invited to a Mediterranean cooking lesson during which they had to produce a complete meal. Individual sessions took place during the first, the sixth and twelfth weeks of the intervention in order to evaluate the dietary changes and to select further objectives for increasing the adherence to the Mediterranean food pattern. During individual sessions, the registered dietitian used FFQ and the Mediterranean food pyramid to identify specific objectives for each participant and to promote and personalise dietary changes to be undertaken. Unannounced qualitative $24 \mathrm{~h}$ recalls were performed by telephone at weeks $2,4,8$ and 10 . The objective of these recalls was to provide support and to reinforce the key principles of the Mediterranean diet. Three registered dietitians were trained to provide a standardised intervention.

\section{$3 d$ Food record}

Each participant completed a $3 \mathrm{~d}$ food record, on two weekdays and one weekend day, at weeks 0,6 and 12 . At the screening of the nutritional intervention (week 0 ), a dietitian provided $15 \mathrm{~min}$ of instruction to each participant on how to complete the food records. Copies of record examples were also provided to each subject. In addition, the participants were encouraged to consume the usual amount of typical foods and drinks. Participants were not required to weigh foods but were asked to measure the volume of the foods consumed with household measurements (cups, tablespoons) or to indicate the weight of commercial products when it was possible to assess portion sizes. The food record included a section for recording information recipes. After completing the food record, the participants met with the dietitian to review all the information for accuracy and completeness and the dietitian used an example of portion size if the portion needed to be more clearly determined.

\section{Mediterranean dietary score}

An administered FFQ was completed at screening (time 0) and then at weeks 6 and 12 of the nutritional intervention. The FFQ was previously described by Goulet et al. (2003). Briefly, the FFQ was administered by a registered dietitian and is based on typical foods available in Québec. It contains ninety-one items and thirty-three subquestions. Participants were questioned about their frequency of intake for different foods during the last month and were asked to report the frequency of these intakes in terms of day, week or month. The diet of the women eligible for the present study had to be typical of the Canadian diet. Accordingly, a partial score varying from 0 to 4 was attributed to each of the eleven components of the pyramid. Components of the Mediterranean pyramid are: grains; fruits; vegetables; legumes, nuts and seeds; olive oil; dairy 
products; fish; poultry; eggs; sweets; red meat and processed meat. The total dietary score could therefore vary between 0 and 44 points (Goulet et al. 2003). Eligible women were those with a score at screening that was below an arbitrary value of 27 .

\section{Anthropometry}

At weeks 0, 6 and 12, body weight, waist circumference and height were measured according to the procedures recommended at the Airlie conference on the standardisation of anthropometric measurements (Lohman et al. 1988) and BMI was calculated.

\section{Plasma lipid and lipoprotein profile}

Blood samples were collected at weeks 0, 6 and 12 from an antecubital vein into vacutainer tubes containing EDTA after a $12 \mathrm{~h}$ overnight fast for the measurements of plasma lipid and lipoprotein concentrations. Total plasma cholesterol and TG levels were determined by enzymatic methods by using the Technicon RA-500 analyzer (Bayer Corp Inc., Tarrytown, NY, USA), as previously described (Moorjani et al. 1986). HDL-C levels were also obtained using an autoanalyser after precipitation of VLDL and LDL in the infranatant fraction with heparin and $\mathrm{MnCl}_{2}$ (Burstein \& Samaille, 1960). Apo B was measured by nephelometry (BN ProSpec; Dade Behring Inc., Newark, NJ, USA) with reagents provided by this company ( $\mathrm{N}$ antisera to human apo B). LDL-C was obtained by the equation of Friedewald et al. (1972). All participants had plasma TG levels below $4.5 \mathrm{mmol} / \mathrm{l}$.

\section{Low-density lipoprotein particle size characterisation}

LDL-PPD and the levels of cholesterol in the small (LDL$\mathrm{C}_{<255 \AA}$ ) and large LDL (LDL-C $>260 \AA$ ) subfractions were obtained by non-denaturing $2-16 \%$ polyacrylamide gel electrophoresis of whole plasma as previously described by St-Pierre et al. (2001). LDL size was computed from the relative migration of four plasma standards of known diameter (Tchernof et al. 1996). The estimated diameter for the major peak in each scan was identified as the LDL-PPD. A mean LDL particle size was also computed by using a modification of the approach described by Tchernof et al. (1996). This integrated LDL particle size corresponded to the weighted mean size of all LDL subclasses in one individual. The relative proportion of LDL with a diameter $<255 \AA$ (LDL \%<255 ${ }^{\circ}$ ) was obtained by computing the relative area of the densitometric scan $<255 \AA$. The absolute concentration of cholesterol in the LDL subfraction with a diameter $<255 \AA$ (LDL-C $<255 \AA)$ was estimated by multiplying the total plasma LDL-C concentrations by the relative proportion of LDL with a diameter $<255 \AA$ as described previously (St-Pierre et al. 2001). A similar approach was used to assess the relative and absolute concentrations of cholesterol in particles with a diameter $>260 \AA \quad(L D L \quad \%>260 \AA$ and LDL$\mathrm{C}_{>260 \AA}$, respectively). Therefore, it has to be emphasised that values of $\mathrm{LDL}-\mathrm{C}_{<255 \AA}$ and $\mathrm{LDL}-\mathrm{C}_{>260 \AA}$ are not direct measurements of cholesterol concentrations in these LDL subfractions.

\section{Nutritional analysis}

The evaluation of nutrient intakes derived from the food records was performed using the Nutrition Data System for Research software (version 4.03; Nutrition Coordination Center, University of Minnesota, Minneapolis, MN, USA) and Food and Nutrient Database 31, released in November 2000 (Schakel et al. 1988). This database includes more than 16000 food items for which the complete nutritional value of 112 nutrients is included. Intakes from vitamin and mineral supplements were not included in the present analysis, which focused on dietary nutrients only.

\section{Statistical analysis}

Data collected at the beginning (week 0) and after 6 and 12 weeks of the intervention were compared using ANOVA for repeated measures to identify time effects. In the presence of significant time effects, contrast analyses were used to determine precisely the location of the significant differences. Since the intervention was conducted over two phases, terms reflecting the potential phase-effect were systematically entered in each model to account for potential interaction with the treatment effect. Spearman correlations were computed to quantify associations among parametric and non-parametric variables. Partial Spearman correlations were performed between changes in LDL electrophoretic characteristics and changes in dietary score to partial out the effect of anthropometric changes. TG concentrations were not normally distributed and analyses were performed on log-transformed values. The dietary variables were log-transformed when necessary to obtain a normal distribution.

Additional analyses were undertaken to determine whether the lipid and lipoprotein response to the intervention might be modulated by baseline LDL size phenotype. In these analyses, participants were divided on the basis of baseline LDL-PPD using tertiles of the distribution (258.4 $\AA$ and 260.0 $\mathrm{A}$ ). The intervention effect within each subgroup as well as the interaction between time and baseline characteristics were calculated by ANOVA. In the presence of significant effects, contrast analyses were performed. All analyses were performed with the SAS statistical package (version 8.02; SAS Institute, Inc., Cary, NC, USA).

\section{Results}

Anthropometric and metabolic profiles of the study participants are presented in Table 1. At 6 weeks after the beginning of the nutritional intervention, total cholesterol, apo $\mathrm{B}$ and BMI decreased slightly but significantly $(P<0 \cdot 01)$. Also, decreases in apo B and BMI remained statistically significant at week 12 but the decrease in plasma total cholesterol did not. Moreover, after 12 weeks of intervention waist circumference was statistically lower than the value measured at baseline $(P<0 \cdot 0001)$. LDL-C, HDL-C and 
Table 1. Anthropometric variables and plasma lipid and lipoprotein profiles at weeks 0,6 and 12 of the nutritional intervention $(n 71) \ddagger$ (Mean values and standard deviations)

\begin{tabular}{|c|c|c|c|c|c|c|}
\hline & \multicolumn{2}{|c|}{ Week 0} & \multicolumn{2}{|c|}{ Week 6} & \multicolumn{2}{|c|}{ Week 12} \\
\hline & Mean & SD & Mean & SD & Mean & SD \\
\hline Age (years) & $46 \cdot 9$ & $7 \cdot 6$ & - & & - & \\
\hline $\mathrm{BMI}\left(\mathrm{kg} / \mathrm{m}^{2}\right)$ & $25 \cdot 8$ & 4.0 & $25 \cdot 6^{\star \star}$ & 4.0 & $25 \cdot 6^{\star \star}$ & 3.8 \\
\hline Waist circumference (mm) & 833 & 109 & 829 & 105 & $821 \dagger$ & 102 \\
\hline Triacylglycerols $\S(\mathrm{mmol} / \mathrm{l})$ & 1.20 & 0.48 & $1 \cdot 18$ & 0.53 & $1 \cdot 16$ & 0.49 \\
\hline Apo B $(g / l)$ & 0.98 & 0.20 & $0.93^{\star \star \star}$ & 0.22 & $0.94^{*}$ & 0.22 \\
\hline Total cholesterol $(\mathrm{mmol} / \mathrm{l})$ & $5 \cdot 21$ & 0.89 & $5 \cdot 08^{\star \star}$ & 0.84 & $5 \cdot 14$ & 0.86 \\
\hline HDL-cholesterol (mmol/l) & 1.64 & 0.41 & 1.59 & 0.39 & 1.62 & 0.41 \\
\hline LDL-C $(\mathrm{mmol} / \mathrm{l}) \|$ & 3.02 & 0.76 & 2.94 & 0.72 & $2 \cdot 97$ & 0.71 \\
\hline LDL-C $<255 \AA(\mathrm{mmol} / \mathrm{l})$ & 0.72 & 0.58 & 0.72 & 0.56 & 0.72 & 0.51 \\
\hline LDL-C $255-260 \AA(\mathrm{mmol} / \mathrm{l})$ & 1.00 & 0.36 & 0.97 & 0.37 & 0.98 & 0.39 \\
\hline LDL-C $>260 \AA(\mathrm{mmol} / \mathrm{l})$ & 1.29 & 0.65 & 1.24 & 0.63 & 1.26 & 0.63 \\
\hline \multicolumn{7}{|l|}{ Relative proportion of LDL (\%) } \\
\hline$<255 \AA$ & 24.0 & $16 \cdot 4$ & 24.8 & $18 \cdot 2$ & 24.7 & $16 \cdot 4$ \\
\hline $255-260 \AA$ & 33.7 & $10 \cdot 0$ & 33.3 & $10 \cdot 8$ & $33 \cdot 3$ & $10 \cdot 8$ \\
\hline$>260 \AA$ & $42 \cdot 3$ & $16 \cdot 4$ & 41.6 & $16 \cdot 6$ & 41.9 & 16.4 \\
\hline LDL peak particle size $(\AA)$ & 258.9 & 2.6 & $258 \cdot 9$ & 2.7 & $259 \cdot 1$ & 2.5 \\
\hline LDL integrated (mean) size $(\AA)$ & $259 \cdot 7$ & $2 \cdot 2$ & 259.6 & $2 \cdot 4$ & 259.6 & $2 \cdot 2$ \\
\hline
\end{tabular}

LDL-C, LDL-cholesterol.

Mean value was significantly different from that at week $0:{ }^{*} P<0.05,{ }^{* *} P<0.01,{ }^{* * *} P<0.0001$.

† Mean value was significantly different from those at weeks 0 and $6(P<0.001)$.

†For details of procedures, see pp. 286-287.

$\S$ Analyses were performed on log-transformed values.

|| Total plasma LDL-C levels were obtained with the Friedewald formula.

TG were not affected significantly by the nutritional intervention. Furthermore, among the total sample of women, no significant changes in LDL-PPD, LDL integrated size, and in the LDL-C distribution among subclasses were observed in response to the nutritional intervention.

As shown in Table 2, the Mediterranean score increased significantly after 12 weeks of nutritional intervention $(21.1$ (SD 3.6) to 29.0 (SD 4.4)). Also, as shown in Fig. 1, 12 weeks after the beginning of the intervention, an increase in the consumption of olive oil, fruits, vegetables, legumes, nuts and seeds, poultry and fish was observed and was reflected by a significant increase of the individual score for these components of the Mediterranean pyramid $(P<0 \cdot 01)$. In addition, a decrease in the consumption of red meat and processed meat and also sweets was observed; this was reflected by significant increases $(P<0.0001)$ in the individual scores for these components of the Mediterranean pyramid.

Table 2 shows the effect of the nutritional intervention on dietary variables as measured by the $3 \mathrm{~d}$ food record.

Table 2. Mediterranean score and daily intake of energy and selected nutrients at weeks 0 and 12 of the nutritional intervention $(n 71) \dagger$

(Mean values and standard deviations)

\begin{tabular}{|c|c|c|c|c|}
\hline & \multicolumn{2}{|c|}{ Week 0} & \multicolumn{2}{|c|}{ Week 12} \\
\hline & Mean & SD & Mean & SD \\
\hline Mediterranean dietary score (arbitrary units) & $21 \cdot 1$ & 3.6 & $29 \cdot 0^{\star \star \star}$ & 4.4 \\
\hline Energy (kJ) & 8590 & 2188 & $7888^{\star *}$ & 1793 \\
\hline Proteins (\%) & $16 \cdot 4$ & 2.5 & $17 \cdot 7^{\star \star}$ & $2 \cdot 9$ \\
\hline Lipids (\%) & $33 \cdot 6$ & $6 \cdot 3$ & 32.4 & $6 \cdot 2$ \\
\hline SFA (\%) & $11 \cdot 2$ & $2 \cdot 8$ & $9 \cdot 9^{\star \star}$ & $2 \cdot 7$ \\
\hline MUFA (\%) & $14 \cdot 2$ & 3.2 & $14 \cdot 2$ & 3.6 \\
\hline PUFA (\%) & $5 \cdot 6$ & 1.6 & $5 \cdot 8$ & 1.4 \\
\hline Cholesterolł (mg) & $280 \cdot 1$ & $139 \cdot 8$ & $225 \cdot 9^{\star \star}$ & $90 \cdot 4$ \\
\hline EPA (g) & 0.06 & 0.07 & $0.12^{\star \star}$ & 0.13 \\
\hline $\mathrm{DHA}(\mathrm{g})$ & 0.17 & 0.23 & $0.27^{\star}$ & 0.36 \\
\hline MUFA:SFA ratio & 1.32 & 0.36 & $1.52^{\star \star}$ & 0.48 \\
\hline Carbohydrates (\%) & $48 \cdot 8$ & 6.9 & 49.8 & 6.9 \\
\hline Total dietary fibres $(\mathrm{g})$ & 21.9 & 6.4 & $25 \cdot 1^{\star \star}$ & $7 \cdot 7$ \\
\hline Soluble fibres $(\mathrm{g})$ & $7 \cdot 3$ & $2 \cdot 0$ & $8 \cdot 2^{\star *}$ & $2 \cdot 4$ \\
\hline Insoluble fibres (g) & 14.4 & 4.7 & $16 \cdot 7^{\star \star}$ & $5 \cdot 5$ \\
\hline Alcohol (\%) & $3 \cdot 7$ & $3 \cdot 6$ & $3 \cdot 2$ & $3 \cdot 1$ \\
\hline
\end{tabular}

SFA, saturated fatty acids; EPA, eicosapentaenoic acid; DHA, docosahexaenoic acid.

Mean value was significantly different from that at week $0:{ }^{\star} P<0.05,{ }^{\star \star} P<0.01,{ }^{\star \star \star} P<0.0001$.

†For details of subjects and procedures, see Table 1 and pp. 286-287.

$\ddagger$ Analyses were performed on log-transformed values. 


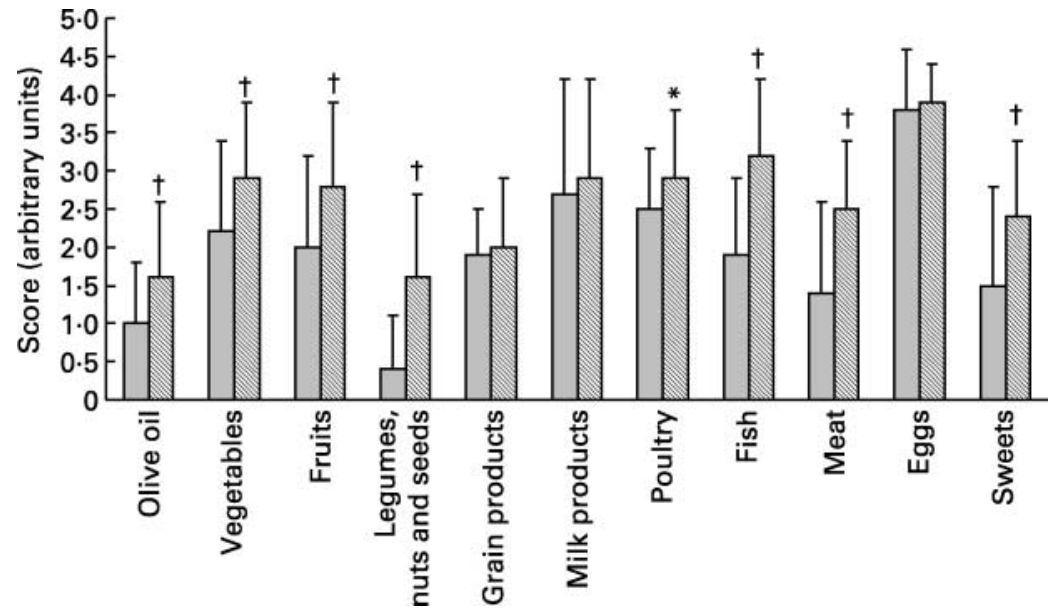

Fig. 1. Individual components of the Mediterranean score at weeks $0(\square)$ and $12(\mathbb{8})$ of the nutritional intervention $(n 71)$. For grains, fruits, vegetables, legumes, nuts and seeds, olive oil, and fish, a high score reflected a high consumption. Inversely for red meat and processed meat, sweets, and eggs, a higher score was attributed for a lower frequency of intake. For dairy products, an intake of two to three portions/d was considered as a typical Mediterranean intake and four points were allowed for such an intake. For poultry, four points were allowed when the mean intake was three portions/week. Individual score was significantly different from that at week $0:{ }^{*} P<0.001, \dagger P<0.0001$.

The 12-week nutritional intervention resulted in significant decreases in energy intake $(745$ (SD 2033) $\mathrm{kJ})$. The relative intake from SFA also significantly decreased from 11.2 (SD $2.8)$ to 9.9 (SD 2.7) \% $(P<0.002)$. A significant increase in the MUFA:SFA ratio was also induced by the nutritional intervention $(P<0.002)$. The relative intake of proteins increased significantly by $1.3 \%$ and the relative intakes of carbohydrates and lipids remained unchanged. A significant increase in the absolute dietary fibre intake was observed $(P<0.01$; Table 2$)$.

Among the total sample of women, increases in the Mediterranean score were significantly associated with decreases in LDL-C concentrations $(r-0.24 ; P=0.04)$. Also, changes in the individual score for sweets were negatively associated with changes in LDL-C $>260 \AA$ concentrations $(r-0.29 ; P=0.02)$, indicating that a decrease in the consumption of sweets was associated with a decrease in LDL-C $>260 \AA$ concentrations. Partial correlation analyses indicated that adjustment for changes in BMI or waist circumference did not modify correlations between changes in items of the Mediterranean score and changes in LDL electrophoretic characteristics.

In our total sample, changes in LDL-PPD were significantly associated with the value of LDL-PPD at baseline $(r-0.43 ; P=0.0002)$. On the basis of this observation, additional analyses were undertaken to investigate the contribution of baseline LDL-PPD values to anthropometric and metabolic changes that occurred during the nutritional intervention. Table 3 shows the baseline characteristics of subjects in each group separated on the basis of LDLPPD at baseline. No significant differences in age, waist circumference and BMI were observed at baseline between the three groups separated on the basis of LDL-PPD. Also, subjects with LDL-PPD $>260 \cdot 0 \AA$ presented at baseline lower concentrations of TG as well as higher HDL-C concentrations than the group with the lowest LDL-PPD $(P<0.05$; Table 3). Differences between groups remained statistically significant at the end of the intervention $(P<0 \cdot 05$; data not shown).
The Mediterranean score increased similarly among the three groups separated on the basis of baseline LDL-PPD (Fig. 2). Also, after 12 weeks of nutritional intervention waist circumference decreased similarly in all groups (Fig. 2) but HDL-C, LDL-C and TG did not change significantly in any of the groups. Furthermore, in response to the nutritional intervention plasma apo B levels decreased significantly after 6 and 12 weeks in subjects with the highest LDL-PPD at baseline $(P<0 \cdot 01 ;$ Fig. 2$)$. In contrast, in subjects characterised by LDL-PPD smaller than $260 \cdot 0 \AA$ (first and second tertiles), apo B concentrations decreased significantly after 6 weeks of nutritional intervention but returned to baseline values after 12 weeks of intervention.

Subjects who, at baseline, were in the first tertile of the LDL-PPD distribution $(<258.4 \AA)$ showed a significant increase in LDL-PPD in response to the 12-week nutritional intervention $(P=0.03$; Fig. 3). Among women with a reduced LDL-PPD at baseline, the proportion of LDL $\%<255 \AA$ tended to decrease $(\mathrm{P}=0 \cdot 12)$ and the proportion of LDL $\%>260 \AA$ increased significantly $(P<0.05)$. In contrast, LDL-PPD and LDL integrated size decreased significantly $(P=0.007)$ among women with large LDL particles at baseline (LDL-PPD $>260 \AA$; Fig. 3). However, in that latter group, LDL-PPD value at the end of the nutritional intervention remained statistically higher than in the two other groups $(P<0 \cdot 0001$; Fig. 3).

\section{Discussion}

The present study conducted in a group of seventy-one healthy women showed that a nutritional intervention promoting the Mediterranean food pattern resulted in significant changes in dietary intakes, which were maintained throughout the 12-week study period. The results also showed that in response to this nutritional intervention, slight but significant improvements in the metabolic profile occurred. Among our total sample, no significant changes in LDL electrophoretic characteristics were observed. However, the present results indicate that beneficial 
Table 3. Baseline characteristics of anthropometric and metabolic variables between the three groups separated on the basis of baseline lowdensity lipoprotein peak particle diameter (LDL-PPD)‡

(Mean values and standard deviations)

\begin{tabular}{|c|c|c|c|c|c|c|}
\hline & \multicolumn{2}{|c|}{$\begin{array}{l}\text { LDL-PPD }_{<258.4 \AA} \\
\quad(n 24)\end{array}$} & \multicolumn{2}{|c|}{$\begin{array}{l}\text { LDL-PPD }_{258.4-260.0 \AA} \\
(n 24)\end{array}$} & \multicolumn{2}{|c|}{$\begin{array}{l}\text { LDL-PPD }_{>260.0 \AA} \\
\quad(n 23)\end{array}$} \\
\hline & Mean & SD & Mean & SD & Mean & SD \\
\hline Age (years) & $45 \cdot 6$ & $9 \cdot 2$ & $45 \cdot 7$ & $5 \cdot 8$ & $49 \cdot 4$ & $7 \cdot 2$ \\
\hline $\mathrm{BMl}\left(\mathrm{kg} / \mathrm{m}^{2}\right)$ & $26 \cdot 4$ & 4.8 & 26.5 & $3 \cdot 8$ & 24.5 & $2 . \overline{9}$ \\
\hline Waist circumference (mm) & 850 & 131 & 851 & 106 & 795 & 79 \\
\hline Triacyglycerols§ (mmol/l) & 1.49 & 0.56 & $1 \cdot 13^{\star}$ & 0.40 & $0.96^{\star}$ & 0.30 \\
\hline Apo B $(g / l)$ & 1.01 & 0.23 & 0.94 & $0 \cdot 16$ & 1.00 & 0.21 \\
\hline Total cholesterol (mmol/l) & 5.04 & 0.92 & $5 \cdot 10$ & 0.85 & 5.54 & 0.85 \\
\hline HDL-cholesterol (mmol/l) & 1.41 & 0.31 & $1.63^{*}$ & 0.37 & $1.89^{\star} \dagger$ & 0.43 \\
\hline LDL-C $\|(\mathrm{mmol} / \mathrm{l})$ & 2.95 & 0.80 & $2 \cdot 92$ & 0.68 & 3.20 & 0.79 \\
\hline LDL-C $<255 \AA(\mathrm{mmol} / \mathrm{l})$ & 0.96 & 0.62 & $0.67^{\star}$ & 0.54 & $0.55^{\star} \dagger$ & 0.50 \\
\hline LDL-C $255-260 \AA(\mathrm{mmol} / \mathrm{l})$ & 1.08 & 0.44 & 1.00 & 0.32 & 0.92 & 0.29 \\
\hline LDL-C $>260 \AA(\mathrm{mmol} / \mathrm{l})$ & 0.90 & 0.43 & $1 \cdot 25^{\star}$ & 0.37 & $1.73^{\star} \dagger$ & 0.79 \\
\hline \multicolumn{7}{|l|}{ Relative proportion of LDL (\%) } \\
\hline$<255 \AA$ & $32 \cdot 1$ & $16 \cdot 5$ & $22 \cdot 1^{*}$ & $14 \cdot 6$ & $17 \cdot 4^{*}$ & $15 \cdot 0$ \\
\hline $255-260 \AA$ & $37 \cdot 2$ & $12 \cdot 1$ & 34.2 & $8 \cdot 1$ & $29 \cdot 6^{*}$ & 8.2 \\
\hline$>260 \AA$ & $30 \cdot 6$ & 11.9 & $43 \cdot 7^{\star}$ & $11 \cdot 3$ & $53 \cdot 0^{*} \dagger$ & $17 \cdot 3$ \\
\hline LDL peak particle size $(\AA)$ & $256 \cdot 1$ & $2 \cdot 0$ & $259 \cdot 2^{*}$ & 0.5 & $261 \cdot 5^{\star} \dagger$ & $1 \cdot 0$ \\
\hline LDL integrated (mean) size $(\AA)$ & 258.0 & 1.9 & $259 \cdot 6^{*}$ & $1 \cdot 2$ & $261 \cdot 7^{\star} \dagger$ & 1.4 \\
\hline
\end{tabular}

LDL-C, LDL-cholesterol.

Mean value was significantly different from that for group $1(P<0.05)$.

† Mean value was significantly different from that for group $2(P<0.05)$.

‡For details of procedures, see pp. 286-287.

$\S$ Analyses were performed on log-transformed values.

\| Total plasma LDL-C levels were obtained with the Friedewald formula.

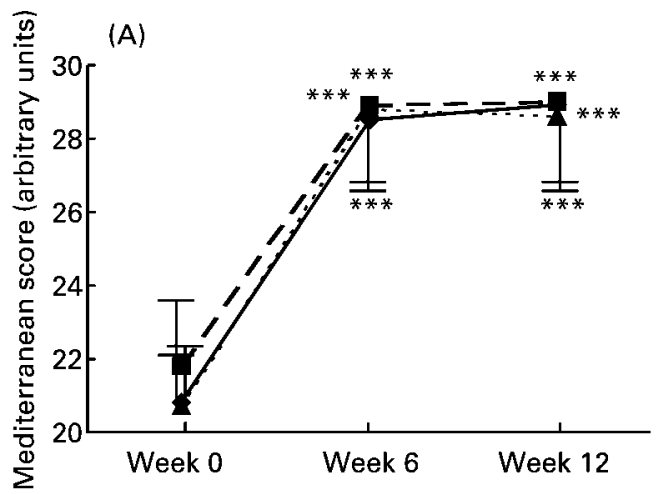

(C)

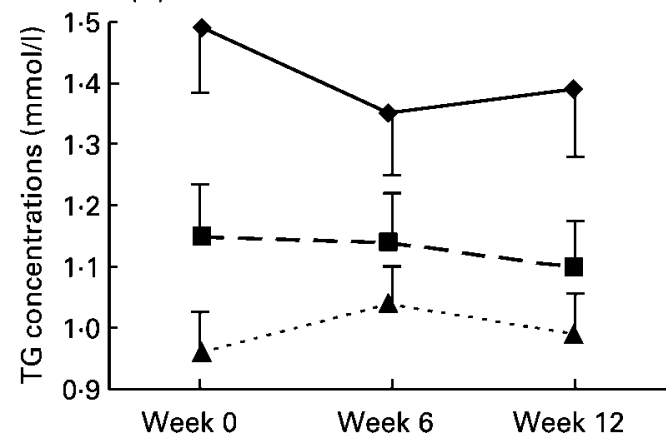

(B)

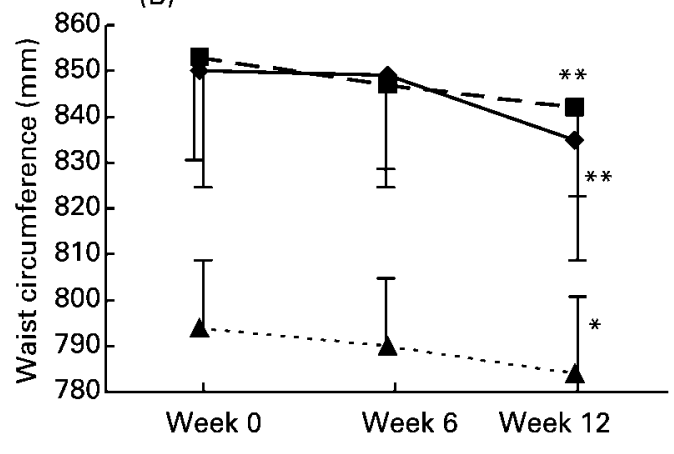

(D)

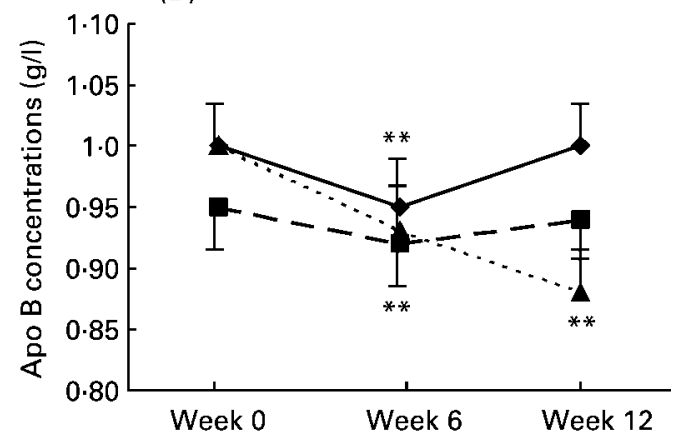

Fig. 2. Impact of LDL peak particle diameter (LDL-PPD) at baseline on response to the nutritional intervention in (A) Mediterranean score, (B) waist circumference, (C) triacylglycerols (TG) concentrations and (D) apo B concentrations. (- -), LDL-PPD <258.4 $\AA$; (-匹-), LDL-PPD

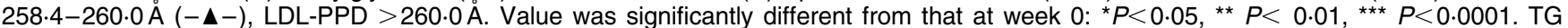
concentrations were significantly different between groups at week $12(P=0 \cdot 05)$. For apo B concentrations, there was a significant interaction $(P=0.02)$. 
(A)

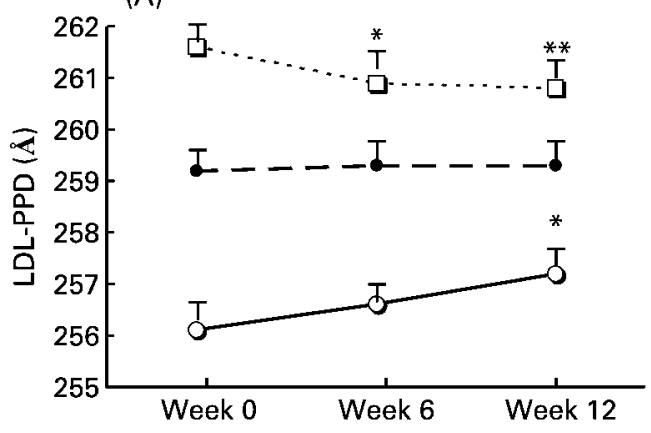

(C)



(B)

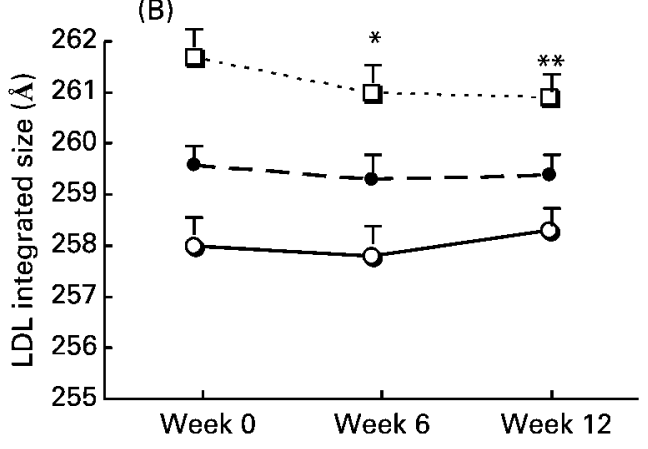

(D)

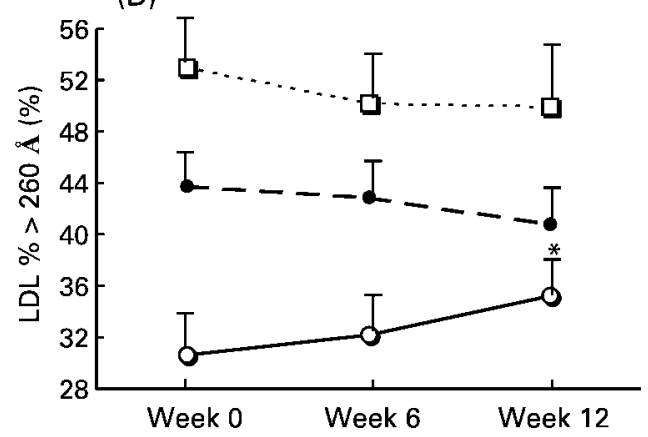

Fig. 3. Impact of LDL peak particle diameter (LDL-PPD) at baseline on response to the nutritional intervention in (A) LDL-PPD, (B) LDL integrated size, (C) LDL \%<255 Value was significantly different from that at week $0:{ }^{*} P<0.05$, ${ }^{*} P<0.01$. Significant differences between groups at week 12 were observed for LDL-PPD $(P<0.0001)$, LDL integrated size $(P<0.001)$ and LDL \%>260 $(P<0.01)$. For LDL-PPD the $P$ for interaction was $P=0.002$, that for LDL integrated size was $P=0.01$, that for LDL $\%<255 \AA$ was $P=0.05$, and that for LDL $\%>260 \AA$ was $P=0.09$.

changes in LDL electrophoretic characteristics occurred among the subgroup of women characterised by smaller LDL particles at baseline (LDL-PPD $<258.4 \AA$ ).

Individuals living in the Mediterranean region are recognised as having one of the lowest rates of mortality from CHD (Keys et al. 1986). Results from two nutritional interventions promoting a Mediterranean diet in secondary prevention indicated that a Mediterranean diet could lead to a significant reduction of recurrence rate of myocardial infarction (de Lorgeril et al. 1994; Renaud et al. 1995; de Lorgeril et al. 1999; Singh et al. 2002). Results of these studies suggest that changes in traditional lipid risk factors such as cholesterol, TG and HDL-C could not explain the reduction of myocardial infarction risk in response to a Mediterranean diet. Since these studies did not succeed in providing clear evidence about the potential mechanisms explaining how the Mediterranean diet would reduce myocardial infarction risk, this indicated that other risk factors could be involved.

Reduced LDL-PPD is usually associated with an increased risk of cardiovascular disease (Austin et al. 1988; Lamarche et al. 1997). It has recently been shown that the relative and absolute proportion of small LDL particles (LDL $\%_{<255 \AA}$; LDL- $\mathrm{C}_{<255 \AA}$ ) is providing valuable additional information to the more traditional measurement of LDL-PPD in predicting CHD risk in men (St-Pierre $e t$ al. 2001). The present results indicate that in our total sample no significant changes in LDL electrophoretic characteristics occurred in response to the 12-week nutritional intervention in healthy women despite significant dietary modifications.
Therefore, it appears that changes in the dietary pattern, taken as a whole, were not accompanied by significant changes in LDL electrophoretic characteristics.

Correlation analysis performed in our total sample demonstrated that the decrease in sweets consumption was significantly associated with a decrease in LDL-PPD. These results suggest that women who decreased the most their consumption of sweets were more likely to have a reduction in LDL-PPD. It is important to specify that many different types of foods were included in the 'sweets' category of the Mediterranean score. In fact, cakes, pies, cookies, chocolate bars, and ice cream were all considered as sweets. Therefore, sweets were not exclusively composed of sugars and it is difficult to specifically identify what nutrients were included in the 'sweets' or what association of nutrients could mediate the association observed between the decrease in sweets consumption and the concurrent reduction in LDL-PPD. Considering this finding, one could argue that a decrease in sweets consumption is not beneficial for cardiovascular health as it is associated with a decrease in LDL-PPD. However, it has to be emphasised that changes in the individual score of sweets tended to be associated with changes in waist circumference $(r-0.21 ; \mathrm{P}=0.07)$ and in LDL-C concentrations $(r-0.27 ; \mathrm{P}<0.02)$. Therefore, a decrease in sweets consumption can favour a waist-circumference reduction and a reduction in LDL-C concentrations that can have long-term beneficial metabolic effects. Also, in contrast to previous studies conducted under isoenergetic conditions (Dreon et al. 1998; Kratz et al. 2002) the present 
study did not report a deleterious effect of a decrease in SFA consumption on LDL electrophoretic characteristics.

When women with smaller LDL particles were analysed separately, some significant changes in LDL electrophoretic characteristics were observed. In fact, women characterised by a more deteriorated risk profile benefited more from the intervention than women presenting a healthier profile at baseline. In women at higher CHD risk, i.e. smaller LDLPPD, higher TG and lower HDL-C, we observed an increase in LDL-PPD and the relative proportion of LDL $\%>260 \AA$ increased while the proportion of LDL $\%<255 \AA$ tended to be reduced. Correlation analyses revealed a negative association between changes in TG concentrations and changes in LDL-PPD in women with smaller LDL particles (data not shown). These results are concordant with the fact that plasma TG is a strong predictor of LDL-PPD (Griffin et al. 1994; Stampfer et al. 1996). It has to be acknowledged that, in the present study, women with smaller LDL particles to start with were still characterised by a metabolic profile that was, for most of them, ranging within normal values. In fact, $75 \%$ of women in the subgroup with smaller LDL particles had LDL-PPD above $255 \AA$ and were therefore characterised by pattern A.

In participants in the third tertile (LDL-PPD > $260 \AA$ ) a decrease in LDL-PPD was observed with no change in the relative distribution of cholesterol among LDL subclasses. Despite a decrease in LDL-PPD in response to our nutritional intervention in these women, LDL-PPD values at the end of the intervention remained relatively high (260.7 (SD 1.3) $\AA$ ) and this decrease probably had no major clinical impact, considering all the other risk factors. In fact, these women responded to the nutritional intervention with a decrease in plasma apo $\mathrm{B}$ concentration and a trend for a decrease in LDL-C concentration and also showed a decrease in waist circumference; these parameters can all have a beneficial impact on CHD risk.

The present results suggest that the change in LDL-PPD in response to the intervention was influenced by the baseline LDL-PPD of our subjects. Differences in the response to the intervention in the three groups separated on the basis of baseline LDL-PPD could not be attributable to differences in dietary changes or changes in waist circumference which were similar between each subgroup in response to our nutritional intervention. Therefore, despite similar dietary changes, the present results suggest that women with smaller LDL-PPD did not respond in the same way to the intervention as the subjects with larger particles. This observation is concordant with previous observations showing that healthy men on a high-fat diet who were characterised by smaller LDL particles at baseline were more responsive to a fat reduction than men with larger particles by showing significant decreases in mass of medium and small particles (Krauss \& Dreon, 1995). Finally, changes observed in the present study confirm that the diet could influence LDL electrophoretic characteristics, but other factors such as genes can have an effect on this phenotype (Bosse et al. 2003). It is also possible that a gene-diet interaction could contribute to the explanation of the inter-individual differences in the effects of the nutritional intervention on the risk of CHD.
In conclusion, a nutritional intervention promoting the Mediterranean food pattern in free-living conditions was effective in modifying the food habits of healthy women from the Québec City metropolitan area. These changes in the food pattern were associated with only marginal variations in LDL electrophoretic characteristics. However, even in a group of healthy women, those in the risk category based on their LDL-PPD experienced significant and beneficial changes in their LDL size phenotype. These subtle changes in their LDL particle size and in cholesterol distribution among various LDL subfractions may explain part of the cardiovascular benefits that have been attributed to the Mediterranean diet in subjects at increased CHD risk. However, it is unclear how these small changes in LDL-PPD in women with smaller LDL particles at baseline could modify the overall cardiovascular risk profile.

We acknowledge that the present study has some limitations. In fact, the lack of a control group can be considered as a limitation. However, we believe that the more important changes in metabolic variables in response to dietary changes that were observed among women with a more deteriorated risk profile at baseline support the thesis that the changes observed are related to the intervention rather than being explained by a study effect. Further studies will be needed to establish whether our intervention is more efficacious in modifying the cardiovascular risk profile, including LDL electrophoretic characteristics, of high-risk individuals than other standard nutritional interventions such as the American Heart Association step I and II diets (National Cholesterol Education Program, 1993).

\section{Acknowledgements}

S. L. is a research scholar from the Fonds de la Recherche en Santé du Québec and B. L is the recipient of a Canada Research Chair in Nutrition, Functional Foods and Cardiovascular Health from the Canada Research Chair Program. The present study was partly supported by the Canada Research Chair in Nutrition, Functional Foods and Cardiovascular Health from the Canada Research Chair Program. The authors express their gratitude to the subjects and their families for their motivation and involvement throughout the study. We acknowledge the contribution of Nancy Gilbert to the nutritional intervention and we thank Danielle Aubin for nursing assistance.

\section{References}

Archer WR, Lamarche B, St-Pierre AC, et al. (2003) High carbohydrate and high monounsaturated fatty acid diets similarly affect LDL electrophoretic characteristics in men who are losing weight. J Nutr 133, 3124-3129.

Austin MA, Breslow JL, Hennekens CH, Buring JE, Willett WC \& Krauss RM (1988) Low-density lipoprotein subclass patterns and risk of myocardial infarction. JAMA 260, 1917-1921.

Bosse Y, Perusse L, Despres JP, Lamarche B, Chagnon YC, Rice T, Rao DC, Bouchard C \& Vohl MC (2003) Evidence for a major quantitative trait locus on chromosome 17q21 affecting low-density lipoprotein peak particle diameter. Circulation 107, 2361-2368. 
Burstein M \& Samaille J (1960) On a rapid determination of the cholesterol bound to the serum $\alpha$ - and $\beta$-lipoproteins (in French). Clin Chim Acta 5, 609-609.

de Lorgeril M, Renaud S, Mamelle N, Salen P, Martin JL, Monjaud I, Guidollet J, Touboul P \& Delaye J (1994) Mediterranean alpha-linolenic acid-rich diet in secondary prevention of coronary heart disease. Lancet 343, 1454-1459.

de Lorgeril M, Salen P, Martin JL, Monjaud I, Delaye J \& Mamelle N (1999) Mediterranean diet, traditional risk factors, and the rate of cardiovascular complications after myocardial infarction: final report of the Lyon Diet Heart Study. Circulation 99, 779-785.

Dreon DM, Fernstrom HA, Campos H, Blanche P, Williams PT \& Krauss RM (1998) Change in dietary saturated fat intake is correlated with change in mass of large low-density-lipoprotein particles in men. Am J Clin Nutr 67, 828-836.

Friedewald WT, Levy RI \& Fredrickson DS (1972) Estimation of the concentration of low-density lipoprotein cholesterol in plasma, without use of the preparative ultracentrifuge. Clin Chem 18, 499-502.

Goulet J, Lamarche B \& Lemieux S (2003) Effect of a nutritional intervention promoting the Mediterranean food pattern on plasma lipids, lipoproteins and body weight in healthy French-Canadian women. Atherosclerosis 170, 115-124.

Griffin BA, Freeman DJ, Tait GW, Thomson J, Caslake MJ, Packard CJ \& Shepherd J (1994) Role of plasma triglyceride in the regulation of plasma low density lipoprotein (LDL) subfractions: relative contribution of small, dense LDL to coronary heart disease risk. Atherosclerosis 106, 241-253.

Keys A, Menotti A, Karvonen MJ, et al. (1986) The diet and 15year death rate in the seven countries study. Am J Epidemiol 124, 903-915.

Kratz M, Gulbahce E, von Eckardstein A, Cullen P, Cignarella A, Assmann G \& Wahrburg U (2002) Dietary mono- and polyunsaturated fatty acids similarly affect LDL size in healthy men and women. $J$ Nutr 132, 715-718.

Krauss RM \& Dreon DM (1995) Low-density-lipoprotein subclasses and response to a low-fat diet in healthy men. $\mathrm{Am}$ $J$ Clin Nutr 62, 478S-487S.

Lamarche B, Tchernof A, Moorjani S, Cantin B, Dagenais GR, Lupien PJ \& Despres JP (1997) Small, dense low-density lipoprotein particles as a predictor of the risk of ischemic heart disease in men. Prospective results from the Quebec Cardiovascular Study. Circulation 95, 69-75.

Lohman T, Roche A \& Martorel R (editors) (1988) The Airlie (VA) consensus conference. In Standardization of Anthropometric Measurements, pp. 39-80. Champaign, IL: Human Kinetics.

Mauger JF, Lichtenstein AH, Ausman LM, Jalbert SM, Jauhiainen M, Ehnholm C \& Lamarche B (2003) Effect of different forms of dietary hydrogenated fats on LDL particle size. Am J Clin Nutr 78, 370-375.

Moorjani S, Gagné C, Lupien PJ \& Brun D (1986) Plasma triglycerides related decrease in high-density lipoprotein cholesterol and its association with myocardial infarction in heterozygous familial hypercholesterolemia. Metabolism 35, 311-316.

Mori TA, Burke V, Puddey IB, Watts GF, O'Neal DN, Best JD \& Beilin LJ (2000) Purified eicosapentaenoic and docosahexaenoic acids have differential effects on serum lipids and lipoproteins, LDL particle size, glucose, and insulin in mildly hyperlipidemic men. Am J Clin Nutr 71, 1085-1094.

National Cholesterol Education Program (1993) Second Report of the Expert Panel on Detection, Evaluation and Treatment of High Blood Cholesterol in Adults (Adult Treatment Panel II) NIH publication no. 93-3095. Bethesda, MD, USA: Department of Health and Human Services, National Institutes of Health, National Heart, Lung and Blood Institute.

Pedersen A, Baumstark MW, Marckmann P, Gylling H \& Sandstrom B (2000) An olive oil-rich diet results in higher concentrations of LDL cholesterol and a higher number of LDL subfraction particles than rapeseed oil and sunflower oil diets. J Lipid Res 41, 1901-1911.

Renaud S, de Lorgeril M, Delaye J, Guidollet J, Jacquard F, Mamelle N, Martin JL, Monjaud I, Salen P \& Toubol P (1995) Cretan Mediterranean diet for prevention of coronary heart disease. Am J Clin Nutr 61, Suppl. 6, 1360S-1367S.

Rivellese AA, Maffettone A, Vessby B, Uusitupa M, Hermansen K, Berglund L, Louheranta A, Meyer BJ \& Riccardi G (2003) Effects of dietary saturated, monounsaturated and n-3 fatty acids on fasting lipoproteins, LDL size and post-prandial lipid metabolism in healthy subjects. Atherosclerosis 167, 149-158.

Schakel SF, Sievert YA \& Buzzard IM (1988) Sources of data for developing and maintaining a nutrient database. $J$ Am Diet Assoc 88, 1268-1271.

Singh RB, Dubnov G, Niaz MA, Ghosh S, Singh R, Rastogi SS, Manor O, Pella D \& Berry EM (2002) Effect of an Indo-Mediterranean diet on progression of coronary artery disease in high risk patients (Indo-Mediterranean Diet Heart Study): a randomised single-blind trial. Lancet 360, 1455-1461.

Stampfer MJ, Krauss RM, Ma J, Blanche PJ, Holl LG, Sacks FM \& Hennekens CH (1996) A prospective study of triglyceride level, low-density lipoprotein particle diameter, and risk of myocardial infarction. JAMA 276, 882-888.

St-Pierre AC, Ruel IL, Cantin B, Dagenais GR, Bernard PM, Despres JP \& Lamarche B (2001) Comparison of various electrophoretic characteristics of LDL particles and their relationship to the risk of ischemic heart disease. Circulation 104, 2295-2299.

Tchernof A, Lamarche B, Prud'Homme D, Nadeau A, Moorjani S, Labrie F, Lupien PJ \& Despres JP (1996) The dense LDL phenotype. Association with plasma lipoprotein levels, visceral obesity, and hyperinsulinemia in men. Diabetes Care 19, 629-637.

Tinker LF, Parks EJ, Behr SR, Schneeman BO \& Davis PA (1999) (n-3) Fatty acid supplementation in moderately hypertriglyceridemic adults changes postprandial lipid and apolipoprotein B responses to a standardized test meal. $J$ Nutr 129, 1126-1134. 